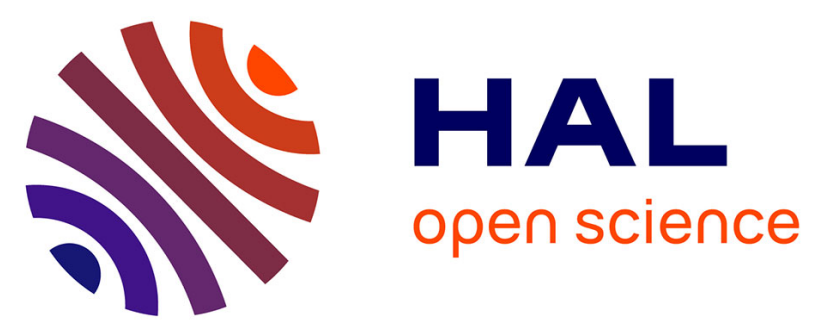

\title{
Adaptability of parabolic wave equation and Gaussian Beam Shooting methods to electromagnetic propagation in urban configurations
}

Arnaud Ginestet, Christine Letrou, Gilles Beauquet, Emna-Amira Fnaiech

\section{- To cite this version:}

Arnaud Ginestet, Christine Letrou, Gilles Beauquet, Emna-Amira Fnaiech. Adaptability of parabolic wave equation and Gaussian Beam Shooting methods to electromagnetic propagation in urban configurations. RADAR 2014: International Radar Conference, Oct 2014, Lille, France. pp.1 - 6, 10.1109/RADAR.2014.7060263 . hal-01255020

\author{
HAL Id: hal-01255020 \\ https://hal.science/hal-01255020
}

Submitted on 11 Feb 2016

HAL is a multi-disciplinary open access archive for the deposit and dissemination of scientific research documents, whether they are published or not. The documents may come from teaching and research institutions in France or abroad, or from public or private research centers.
L'archive ouverte pluridisciplinaire HAL, est destinée au dépôt et à la diffusion de documents scientifiques de niveau recherche, publiés ou non, émanant des établissements d'enseignement et de recherche français ou étrangers, des laboratoires publics ou privés. 


\title{
Adaptability of Parabolic Wave Equation and Gaussian Beam Shooting methods to Electromagnetic Propagation in Urban Configurations
}

\author{
Arnaud Ginestet*, Christine Letrou ${ }^{\dagger}$, Gilles Beauquet ${ }^{\ddagger}$ \\ and Emna-Amira Fnaiech ${ }^{\dagger}$ \\ ${ }^{*}$ NOVELTIS \\ 153 rue du Lac, 31670 Labege, France \\ Email: arnaud.ginestet@noveltis.fr \\ ${ }^{\dagger}$ SAMOVAR (UMR CNRS 5157) \\ Institut TELECOM SudParis \\ 9 rue Charles Fournier, 91011 Evry Cedex, France \\ $\ddagger$ THALES Air Systems S.A. \\ Hameau de Roussigny, 91470 Limours, France
}

\begin{abstract}
In spite of well-known limitations, ray based methods are the quasi-unique tool for modelling the electromagnetic propagation in urban areas. As an alternative to these methods, two models based on the resolution of the Parabolic Wave Equation, and on Gaussian Beam Shooting, are proposed. Both are specifically adapted to meet the challenges of urban areas propagation: three-dimensional, wide angle approximation and considering the backscatter propagation for the first one; taking into account ground and grazing angles for the second one. A preliminary test case is presented and configurations of interest under processing are detailed.
\end{abstract}

\section{INTRODUCTION}

\section{A. Context}

The knowledge of electromagnetic propagation is critical to increase the efficiency and need for control of communication systems. It improves detection and discrimination capabilities of radar systems in presence of dense obstacles.

Nowadays cities become a major configuration, since for the first time in the history of mankind the urban population outnumbered rural population in 2007. More, this ratio is expected to reach $60 \%$ by 2030 . This change has to be taken into account by the scientific community, regardless of their speciality.

Indeed, the presence of obstructing buildings in an urban areas causes shadowing of the emitter from the receiver and can lead to severe loss of signals. Thus, electromagnetic propagation models need to be adapted to the specific urban configurations, as far as three-dimensional processing becomes mandatory.

\section{B. Configurations of interest}

When dealing with electromagnetic propagation, configurations of interest will support the efficiency assessment of modelling tools regarding two objectives:
1) Analysis of urban areas and semi-urban areas as electromagnetic scenes;

2) Production of enough results to help the deployment of a system (radar or stations).

The interest lies in the theoretical forecast of performances as close as possible to reality: it concerns attenuation between Base Station (BS) and User Equipment (UE) for broadband mobile or it concerns complete monitoring of radar detection loss due to obstacles in civil, military or security domains.

Masking and multiple reflections must be taken into account in three-dimensional electromagnetic scenes. Elementary mechanisms in play could be:

- Obstruction due to erected obstacle(s) close to the emitting antenna or close to the receiving antenna;

- Lateral deviation on large distant walls;

- Influence of relief (at a large scale): beam is guided in a turning valley or dispersed around a hill.

Configurations of interest can be illustrated in the two following cases.

1) Microcell: There are a lot of Line-Of-Sight (LOS) and Non-Line-Of-Sight (NLOS) geometrical situations encountered in urban area as shown in Fig. 1. Various multiple propagation mechanisms occur when BS and UE are operating from and close to tall buildings, in particular those relayed by roofs. Table I lists parameters and their ranges when considering typical configurations.

2) ATC radars: A Primary Surveillance Radar (PSR) system is deployed on an airport. The beam set is rotated. The forward way is sought as not obstructed, except for certain beam directions where building or tower can affect the bottom of the radar beam and could also reflect backward a part of energy. For monopulse radar, signal processing can be more 


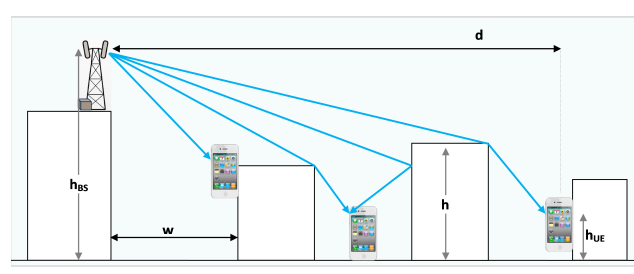

Fig. 1. Configuration of interest no. 1 - Geometry

TABLE I

CONFIGURATION OF INTEREST NO. 1 - PARAMETERS INSPIRED FROM UIT-R P.1411-6 (02/2012)

\begin{tabular}{|c||c|c|c|}
\hline & Description & Unit & Range \\
\hline \hline $\mathrm{h}$ & Height of buildings & {$[\mathrm{m}]$} & 4 to 50 \\
\hline $\mathrm{w}$ & Street width & {$[\mathrm{m}]$} & 10 to 25 \\
\hline$\delta$ & Building density & {$[\%]$} & 20 to 40 \\
\hline$h_{B S}$ & Base Station height & {$[\mathrm{m}]$} & 1 to 100 \\
\hline$h_{U E}$ & User Equipment height & {$[\mathrm{m}]$} & A few \\
\hline $\mathrm{d}$ & Distance BS to UE & {$[\mathrm{m}]$} & 1000 (up to 5000$)$ \\
\hline $\mathrm{f}$ & frequency & {$[\mathrm{MHz}]$} & 800 to 20000 \\
\hline $\mathrm{s}$ & UE speed & {$\left[m . \mathrm{s}^{-1}\right]$} & 0 to 30 \\
\hline
\end{tabular}

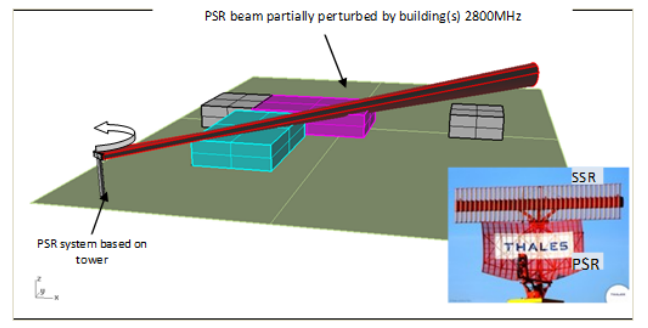

Fig. 2. Configuration of interest no. 2 - Perturbed beam

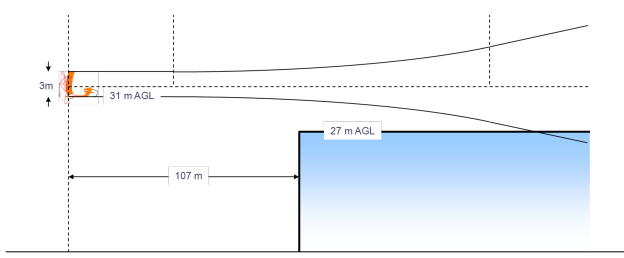

Fig. 3. Configuration of interest no. 2 - Dimensions projected in vertical cut

particularly sensitive to environment through each antenna channel. Accurate calculation requires deep knowledge of real diagrams in presence of the ground and close structure. ATC operation bands are $[1215 \mathrm{MHz}-1370 \mathrm{MHz}]$ in L-band and [2700 MHz-2900 MHz] in S-band. For secondary Surveillance Radar Systems (SSR), uplink runs at $1030 \mathrm{MHz}$ and downlink at $1090 \mathrm{MHz}$. All these systems are designed with very narrow beams: azimuthal beam width could be down to $1.5^{\circ}$. Fig. 2 shows a realistic case where a S-band radar is installed near a hangar: dimensions are given for a vertical cut in Fig. 3. The theoretical flanges of the beam at $3 \mathrm{~dB}$ encounters the flat roof at about $200 \mathrm{~m}$.

\section{Literature review}

The study of electromagnetic wave propagation in urban areas has led to numerous studies, mostly since the advent of mobile telephony. Thus, in reference to the work done within the COST (European Co-operation in the field of Scientific and Technical research) and more precisely the action 231 (Evolution of Land Mobile Radio Communications), a large number of models have been developed. The following models can be highlighted as the most significant ones: the "COSTHata-Model" [1], the "COST-Walfish-Ikegami-Model" [2] [3], the "CNET ray lauching model" [4], the "ASCOM-ETH micro-cell model" [5] [6], the "Villa Griffone's Lab (VGL) model" [7], the "Uni-Stuttgart 3D micro-cell model" [8] [9] [10] and the "Uni-Karlsruhe model 3D-URBAN-MICRO" [11] [12] [13] [14]. However, these models are either empirical models computing only attenuation in two dimensions and consequently neglecting three-dimensional effects; or models based on ray tracing and thus implying the limitations of this method (i.e. very large number of rays and thus unsustainable computation time, presence of caustic(s), far field approximation... specifically in urban configurations).

Our objective is to overcome the limitations of previous models, developing new formulations and new algorithms for modelling the electromagnetic field propagation in urban areas, based on the three-dimensional Parabolic Wave Equation and the three-dimensional Gaussian Beam Shooting.

1) Parabolic Wave Equation: Parabolic Wave Equation (PWE) remains for many years one of the most effective method to simulate electromagnetic propagation in complex environments. However, it is based on two restrictions: the paraxial approximation reducing the validity of the method to a cone around the main propagation direction and the ignoring the back-propagation.

In urban or semi-urban areas, we often encounter the scenario of an obstacle (i.e. a building) obstructing or interfering with the electromagnetic propagation. This will generate reflections on the sides or diffractions by the edges of this obstacle, or in the worst case a back-propagation of the EM signal. Given the limitations of the Parabolic Wave Equation method, urban or semi-urban configurations are disadvantageous using this method.

Nevertheless, it is possible to enlarge the cone of validity of the Parabolic Wave Equation when using a development of higher order (i.e. Feit and Fleck formulation [15]). Recently, a Parabolic Wave Equation resolution taking into account both the forward and backward propagation, called "two-ways", was developed. The idea (behind this resolution) is to consider both directions of propagation by reversing the main direction in the resolution of the Parabolic Wave Equation [16] [17] [18]. In [19], a two-ways finite element resolution is presented for the modelling of ground wave propagation over irregular terrain and in the presence of an inhomogeneous atmosphere.

It is interesting to note that all the two-ways resolutions of the Parabolic Wave Equation currently published are restricted to two-dimensional areas. Our ambition is therefore to implement a three-dimensional wide-angle 2-ways resolution of 
the Parabolic Wave Equation in order to overcome its current limitations and to make it adapted to urban configurations.

2) Gaussian Beam Shooting: Although the Gaussian Beam Shooting (GBS) method is particularly well suited to 3D field simulations in multi-reflecting contexts, few works are currently devoted to the use of Gaussian Beams (GB) to model electromagnetic propagation in urban or semi-urban areas.

In the millimetric range, "simple" versions of Gaussian Beam Shooting have provided results equivalent to that obtained by ray tracing techniques. In these "simple" algorithms, no specific formulation is developed to account for diffraction. If a beam axis is incident on an obstacle, the beam is considered as if incident on an infinite surface. Conversely, if the beam axis does not intercept the obstacle, the beam is considered as if propagating in free space. This methodology was validated by measurements around $60 \mathrm{GHz}$ in indoor environments [20] [23]. An ultra-wide band version of this kind of methodology has been implemented for building areas at non-millimetre frequencies, but to our knowledge result validation has not been published [26].

More accurate simulations of diffracted fields, yet keeping their representation as a sum of Gaussian beams (formulations sometimes called "B2B", beam to beam), is however a prerequisite for an efficient use of Gaussian Beam Shooting in multipath propagation environments at frequencies of conventional communication systems or radar systems. As mentioned above, only few recent studies have focused on this problem, and the resulting formulations depend on the method used to represent the radiated or scattered fields as discrete sums of Gaussian beams: empirical sampling and normalisation at arrival [25], empirical sampling in the angular domain and frame decomposition along the edge [27], frame decomposition and frame based truncation of fields on obstacle surface [21] [22] [24]. The latter approach is used in this work and yields Physical Optics like representation of diffracted fields.

\section{FORMULATION}

\section{A. Parabolic Wave Equation}

The vectorial wave function $\Psi(x, y, z)$ can be obtained as the solution of the following three-dimensional Helmholtz equation:

$$
\left\{\frac{\partial^{2}}{\partial x^{2}}+\frac{\partial^{2}}{\partial y^{2}}+\frac{\partial^{2}}{\partial z^{2}}+k_{0}^{2} n^{2}(x, y, z)\right\} \Psi(x, y, z)=0
$$

Where $n(x, y, z)$ is the refractive index, $k_{0}=2 \pi / \lambda$ is the free space wavenumber ( $\lambda$ is the wavelength), and $x, y$ and $z$ are the longitudinal (range), the transverse and the height coordinates, respectively. $\Psi(x, y, z)$ corresponds to either the electric and magnetic field components for horizontal and vertical polarizations.

Considering that the direction of wave propagation is predominantly along the $+x$-axis paraxial direction, the Parabolic Wave Equation is written by separating rapidly varying phase terms $\left(\Psi(x, y, z)=\exp \left(i k_{0} x\right) u(x, y, z)\right)$. That is, the
Parabolic Wave Equation in terms of the slowly varying amplitude function $u(x, y, z)$ is expressed as follows:

$$
\begin{gathered}
\left\{\frac{\partial}{\partial x}+i k_{0}(1-\sqrt{1+T})\right\}\left\{\frac{\partial}{\partial x}+i k_{0}(1+\sqrt{1+T})\right\} \\
u(x, y, z)=0
\end{gathered}
$$

Where $T=k_{0}^{-2}\left(\partial^{2} / \partial y^{2}+\partial^{2} / \partial z^{2}\right)+n^{2}-1$.

The first and second parts of (2) correspond to the forward and backward propagation waves, respectively. If the backward propagation is ignored (2) reduces to:

$$
\begin{aligned}
\left\{A_{0} \frac{\partial}{\partial x}\right. & +A_{1} \frac{\partial}{\partial x}\left(\frac{\partial^{2}}{\partial y^{2}}+\frac{\partial^{2}}{\partial z^{2}}\right) \\
& \left.+A_{2}+A_{3}\left(\frac{\partial^{2}}{\partial y^{2}}+\frac{\partial^{2}}{\partial z^{2}}\right)\right\}=0
\end{aligned}
$$

with the help of $\sqrt{1+T} \approx\left(a_{0}+a_{1} t\right) /\left(b_{0}+b_{1} t\right)$ approximation; therefore, $A_{0}=b_{0}+b_{1}\left(n^{2}-1\right), A_{1}=$ $b_{1} k_{0}^{-2}, A_{2}=i k_{0}\left(\left(b_{0}-a_{0}\right)+\left(b_{1}-a_{1}\right)\left(n^{2}-1\right)\right)$, and $A_{3}=$ $i k_{0}^{-1}(b 1-a 1)$.

If the angle of propagation measured from paraxial direction is less than $15^{\circ}$, the standard Parabolic Wave Equation is obtained with the help of square root approximation $\sqrt{1+t} \approx$ $1+t / 2$. If the propagation angle is more than $15^{\circ}$, the Claerbout equation is obtained by using the first-order Padé approximation $\sqrt{1+t} \approx(1+0.75 t) /(1+0.25 t)$ to satisfy propagation angles up to $40^{\circ}$. Thus, the contribution of a wideangle resolution can be investigated.

A Finite-Difference methodology is then adopted in order to "introduce" the Leontovich boundaries condition into the propagation algorithm.

A comparison between the mixed-implicit scheme and an alternating method will be performed and the contribution of a 2-ways resolution will be quantified.

\section{B. Gaussian Beam Shooting}

In Gaussian Beam Shooting (GBS) algorithms, propagated fields are represented as a superposition of Gaussian beams (GB) launched from the emitting antenna and transformed through successive interactions with obstacles. The formulation developed in this work is based on frame decomposition of source fields:

- The planar distribution of source fields is expressed as a weighted sum of Gaussian windows which form a "frame".

- Each Gaussian window radiates in the form of a Gaussian beam.

1) Frame of Gaussian windows: The frame windows $\psi_{\boldsymbol{\mu}}(y, z)$ used to describe a planar source distribution in the $y O z$ plane are constructed as the product of frame windows $\psi_{m, n}^{y}(y)$ and $\psi_{p, q}^{z}(z)$ in $L_{2}(\mathbb{R})$ :

$$
\psi_{\boldsymbol{\mu}}(y, z)=\psi_{m, n}^{y}(y) \psi_{p, q}^{z}(z)
$$

where $\boldsymbol{\mu}=(m, n, p, q)$ is a composite translation index in $\mathbb{Z}^{4}$. The frame windows $\psi_{m, n}^{y}(y)$ are obtained by translation of 
a Gaussian function $\psi(y)$ along the spatial coordinate $y$ and along its spectral counterpart $k_{y}$ :

$$
\psi_{m, n}^{y}(y)=\psi(y-m \bar{y}) e^{i n \bar{k}_{y} y}
$$

- $\bar{y}$ and $\bar{k}_{y}$ are respectively the spatial and spectral translation steps, $m$ and $n$ the spatial and spectral translation indices.

- $\left\{\psi_{\boldsymbol{\mu}}, \mu \in \mathbb{Z}^{2}\right\}$ is a frame if and only if $\bar{y} \bar{k}_{y}=2 \pi \nu$, with $\nu<1$ ( $\nu$ : oversampling factor).

- the favorite choice for the translation steps is: $\bar{y}=\sqrt{\nu} L$, $\bar{k}_{y}=\sqrt{\nu}(2 \pi / L)$ ("balanced" frame).

The same construction is used for the frame along $z$, with $p$, $q$ the spatial and translation indices along $z$. In the following, the Gaussian function $\psi$ is taken as:

$$
\psi(y)=(\sqrt{2} / L)^{\frac{1}{2}} e^{-\pi\left(\frac{y}{L}\right)^{2}}
$$

2) Frame window radiation: Through paraxial asymptotic evaluation of plane wave spectrum integrals, the fields radiated by the source field distributions $\psi_{\boldsymbol{\mu}}(y, z)$ (either for $y$ or $z$ component) are put in the form of paraxial Gaussian beams. Let us denote $\boldsymbol{B}_{\boldsymbol{\mu}}(\boldsymbol{r})$ the field radiated at point $\boldsymbol{r}$. The paraxial expression of $\boldsymbol{B}_{\boldsymbol{\mu}}$ is:

$$
\boldsymbol{B}_{\boldsymbol{\mu}}(\boldsymbol{r})=\boldsymbol{B}_{0} \sqrt{\frac{\operatorname{det} \boldsymbol{\Gamma}^{-1}(0)}{\operatorname{det} \boldsymbol{\Gamma}^{-1}\left(x_{\boldsymbol{\mu}}\right)}} \exp i k\left[x_{\boldsymbol{\mu}}+\frac{1}{2} \boldsymbol{y}_{\boldsymbol{\mu}}^{t} \boldsymbol{\Gamma}\left(x_{\boldsymbol{\mu}}\right) \boldsymbol{y}_{\boldsymbol{\mu}}\right]
$$

with $\boldsymbol{B}_{0}$ a vector depending on the source polarization. This expression is analog to that of a geometrical optics ray along the $x_{\boldsymbol{\mu}}$ direction, with $\boldsymbol{\Gamma}$ the curvature matrix and $\sqrt{\frac{\operatorname{det} \boldsymbol{\Gamma}^{-1}(0)}{\operatorname{det} \boldsymbol{\Gamma}^{-1}\left(x_{\boldsymbol{\mu}}\right)}}$ the divergence factor. $\boldsymbol{y}_{\boldsymbol{\mu}}=\left(y_{\boldsymbol{\mu}}, z_{\boldsymbol{\mu}}\right)$ is the position vector of the point $r$ in a plane transverse to the $x_{\mu}$ axis.

The difference between geometrical optics rays and Gaussian beams comes from the fact that $\Gamma$ is a real matrix in the first case, a complex one in the latter. This complex matrix accounts for the Gaussian decay of fields with increasing distance from the $x_{\mu}$ axis in transverse planes. The $x_{\boldsymbol{\mu}}$ axis is then called the beam axis.

3) Gaussian beam tracking in a built environment: In the presence of "infinite" smooth interfaces described either as dielectric interfaces or by surface impedance models, GB can be tracked in a way similar to rays, thanks to their paraxial properties. In the case of planar interfaces, reflected or refracted beams can even be expressed as beams originating in "image" source distributions. The presence of ground is easily accounted for in this way. Yet, paraxial beam tracking cannot address problems where beams impinge on discontinuities, as shown in Fig. 4. Specific B2B algorithms are thus developed to simulate propagation in the presence of buildings:

- selection of beams contributing (above a given threshold level) to the incident field distribution on a finite reflecting surface (wall);

- for the selected beams impinging on a building corner, decomposition of their incident fields on a frame of

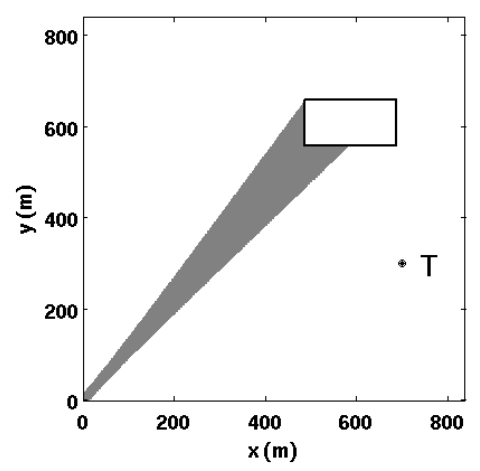

Fig. 4. Gaussian beam impinging on a building corner: 2D cut for a beam propagating in the $x O y$ plane, launched from a frame window source centered at the origin in the $y O z$ plane $(7.36 \mathrm{~m}$ beam width at $430 \mathrm{MHz})$. "T" denotes the target.

narrow windows, which is truncated to the edge of the wall;

- change of frame, yielding the frame coefficients of the field of interest on a set of (spatially) wide windows, which are used to further propagate fields in the form of collimated beams.

Analytical expressions have been established for incident beam field decomposition on a narrow window frame, and for frame change matrix elements [22], [24]. They are under generalization in the case of successive diffractions around the corners of a building.

\section{NUMERICAL RESULTS}

\section{A. Source modelling}

Meaningful comparisons between the Parabolic Wave Equation (PWE) and the Gaussian Beam Shooting (GBS) methods are achievable only when the codes propagate the same source field. For the numerical simulations reported in this article, we define an $O x y z$ coordinate system, wherein $z$ is the vertical axis, the ground is in the plane $x 0 y$ defined by $z=0$, the source is located in the plane $y O z$ defined by $x=0$.

Vertical $\left(E_{y}=0\right)$ and Horizontal $\left(E_{z}=0\right)$ polarizations are studied separately. The non-zero component of the source field, denoted as $E(y, z)$, is taken as:

$$
\begin{aligned}
E(y, z)= & B_{0} \exp \left[-\pi / L_{0}^{2}\left(y^{2}+(z-h)^{2}\right)\right. \\
& \left.\exp i\left(n \bar{k}_{1} y+q \bar{k}_{2} z\right)\right]
\end{aligned}
$$

Where:

- $B_{0}=\sqrt{2} / L_{0}$

- $h$ is the emitter height, defined as $h=p \bar{z}$ with $p \in \mathbb{Z}^{2}$ and $\bar{z}=\sqrt{\nu} L_{0}$;

- $\bar{k}_{1}=\bar{k}_{2}=\sqrt{\nu} 2 \pi / L_{0}$;

- $n$ and $q$ are in $\mathbb{Z}^{2}$ and define the beam pointing respectively along the $y$ and $z$ directions.

For the results presented below, the following values have been used:

- the frequency $f=430 \mathrm{MHz}$; 


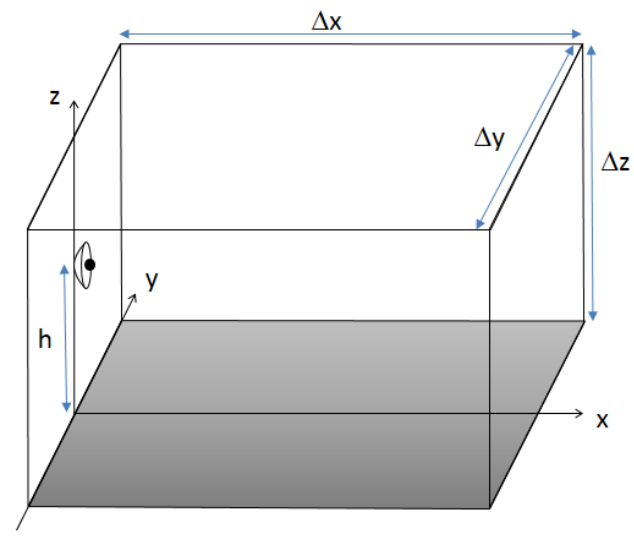

Fig. 5. Test case representation

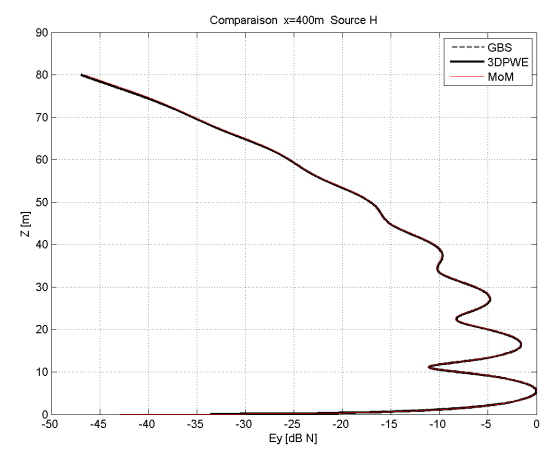

Fig. 6. Comparison between PWE and GBS along $z$, for $y=0 \mathrm{~m}$ and $x=400 \mathrm{~m}$. Validation by comparison with the Method of Moments

- $L_{0}=7.5 \lambda$;

- $\nu=0.16$

- $p=6$.

With these parameters, the source is centered at a height $h$ approximately equal to $12.5 \mathrm{~m}$.

\section{B. Preliminary test case}

A preliminary test case is presented in Fig. 5, with the previously defined source. The polarization is horizontal and the ground is considered flat and perfectly conducting.

Simulation results obtained by both PWE and GBS methods are shown in Fig. 6. The field magnitude is represented along the vertical axis defined by $y=0 \mathrm{~m}$ (laterally centered) and $x=400 \mathrm{~m}$ (distance from the source plane).

We can see an excellent agreement between both methods. Due to the Finite Difference methodology used, PWE results depend on spatial domain sampling (mainly the transverse ones, i.e. in $y$ and $z$ dimensions). Thiner the sampling is, better the modelling is. As an example, the presented result has been obtained thanks to a $\lambda / 10$ transverse sampling.

Validation by comparison with Method of Moments (MoM) [28] is also presented in Fig. 6. Due to the perfect comparison, both methods implemented can be considered as validated on this test case.

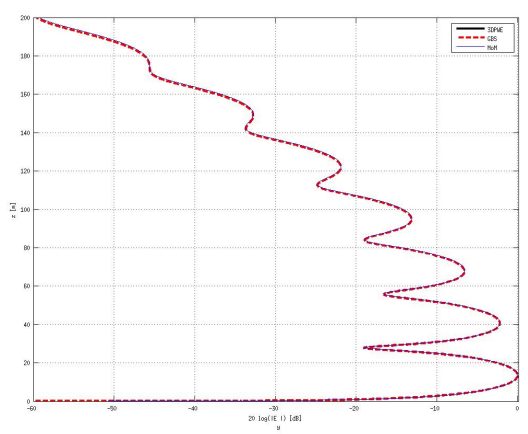

Fig. 7. Comparison between PWE and GBS along $z$, for $y=0 \mathrm{~m}$ and $x=1000$ m. Validation by comparison with the Method of Moments

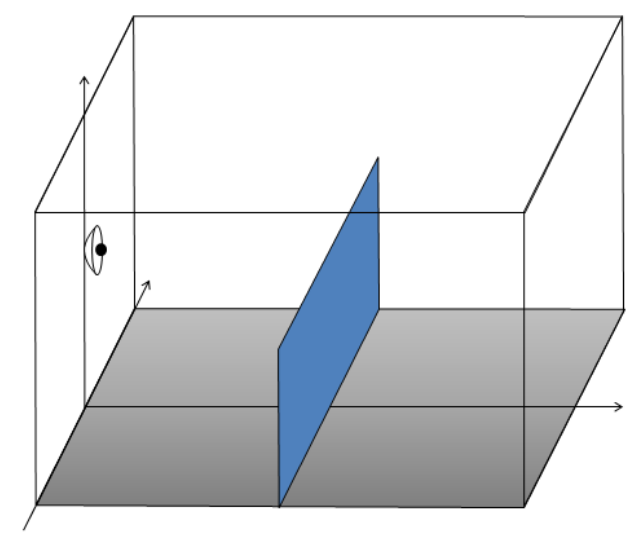

Fig. 8. Screen test case

Comparison between both methods and validation with MoM at a longer distance, i.e. $1 \mathrm{~km}$, is presented in Fig. 7.

Agreement between all methods can still be considered as excellent.

\section{FURTHER WORK}

The first test case was presented, comparing the electromagnetic wave propagation over a perfectly conducting flat ground (at a distance of $400 \mathrm{~m}$ and $1 \mathrm{~km}$ ), modelled by the Parabolic Wave Equation and the Gaussian Beam Shooting methods.

Comparisons on all components of the electromagnetic field are under progress.

The specific developments needed for each method, in order to cope with the configurations of interest presented above, are under test.

More complex test case results (cf. Fig. 8, 9 and 10), and further applied in configurations of interest (cf. Fig. I and 2), will be presented at the conference.

\section{ACKNOWLEDGMENT}

The authors acknowledge partial support by the French National Research Agency (ANR) under "Specific Support for Research Works and Innovation Defense" (ASTRID) grant ANR-12-ASTR-0013. 


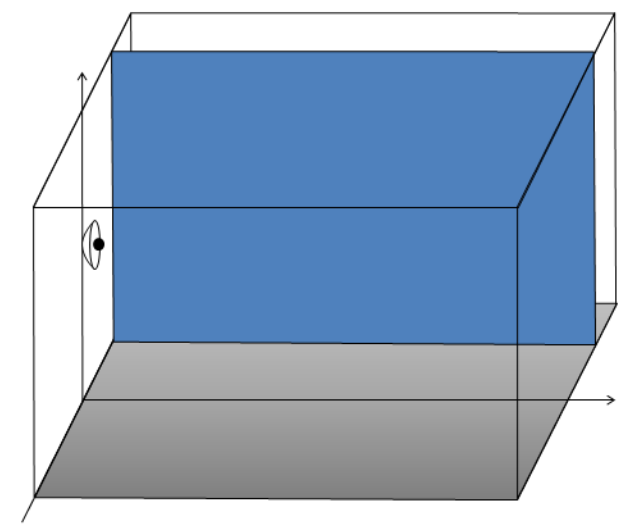

Fig. 9. Lateral wall test case

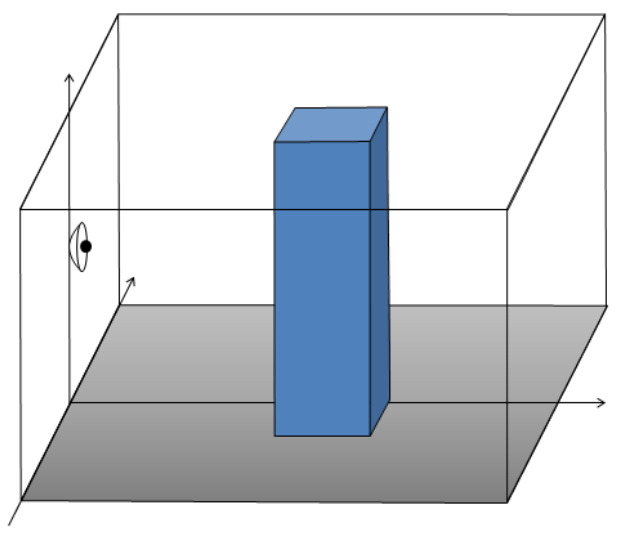

Fig. 10. Isolated building test case

\section{REFERENCES}

[1] Y. Okumura, E. Ohmori, T. Kawano, K. Fukuda, Field strength and its variability in VHF and UHF land-mobile service, Review of the Electrical Communication Laboratory, vol. 16, no. 9-10, pp. 825-873, 1968.

[2] J. Walfisch, H. L. Bertoni, A theoretical model of UHF propagation in urban environments, IEEE Trans. on Antennas and Propagation, vol. 36, no. 12, pp. 1788-1796, December 1988.

[3] F. Ikegami, S. Yoshida, M. Umehira, Propagation factors controlling mean field strength on urban streets, IEEE Trans. on Antennas and Propagation, vol. 32, no. 8, pp. 822-829, August 1984.

[4] J. P. Rossi, J. C. Bic, A. J. Lvy, A ray Shooting model in urban area, COST 231 TD(90)78, Paris, France, October 1990.

[5] U. Dersch, E. Zollinger, Physical characteristics of urban micro-cellular propagation, IEEE Trans. on Antennas and Propagation, vol. 42, no. 11, pp. 1528-1539, November 1994.

[6] U. Dersch, E. Zollinger, Propagation mechanisms in microcell and indoor environments, IEEE Trans. on Vehicular Technology, vol. 43, no. 4, pp. 1058-1066, November 1994.

[7] P. Daniele, V. Degli-Esposti, G. Falciasecca, G. Riva, Field prediction tools for wireless communications in outdoor and indoor environments, Proc. IEEE MTTSymp. European Topical Congress "Technologies for Wireless Applications," Turin, Italy, November 1994.

[8] B. E. Gschwendtner, Adaptive Wellenausbreitungsmodelle fr die Funknetzplanung (in German, Adaptive propagation models for radio network planning), Ph.D. Thesis, University of Stuttgart, ISBN 3-8265-1318-5, 1995.

[9] B. E. Gschwendtner, G. Wlfle, B. Burk, F. M. Landstorfer, Ray tracing vs. ray Shooting in 3-D microcell modellin", Proc. European Personal and Mobile Communications Conference EPMCC95, Bologna, Italy, November 24-26, 1995, pp. 74-79.
[10] B. E. Gschwendtner, F. M. Landstorfer, 3-D propagation modelling in microcells including terrain effects, Proc. IEEE Intern. Personal, Indoor and Mobile Radio Conference PIMRC95, USA, 1995, pp. 532-536.

[11] D. J. Cichon, Strahlenoptische Modellierung der Wellenausbreitung in urbanen Mikro- und Pikofunkzellen (in German, Ray optical modelling of wave propagation in urban micro- and pico-cells), $\mathrm{Ph} . \mathrm{D}$. Thesis, University of Karlsruhe, published in Forschungsberichte aus dem Institut fr Hchstfrequenztechnik und Elektronik der Universitt Karlsruhe, vol. 8, ISSN 09422935, 1994.

[12] Th. Krner, D. J. Cichon, W. Wiesbeck, Concepts and results for $3 D$ digital terrain based wave propagation models - an overview, IEEE Journal on Selected Areas in Communications, vol. 11, no. 7, pp. 10021012, 1993.

[13] D. J. Cichon, T. Krner, W. Wiesbeck, "Modellierung der Wellenausbreitung in urbanem Gelnde (in German, Wave propagation modelling in urban area), FREQUENZ, vol. 47, no. 1-2, pp. 2-11, 1993

[14] D. J. Cichon, W. Wiesbeck, Comprehensive ray optical propagation models for indoor and outdoor environments: Theory and applications, Proc. COMMSPHERE95, Eilat, Israel, January 22-27, 1995, pp. 201-208.

[15] M. D. Feit, J. A. Fleck, Light propagation in graded-index fibers, Applied Optics, vol. 17, pp. 3990-3998, 1978.

[16] O. Ozgun, Recursive two-way parabolic equation approach for modelling terrain effects in tropospheric propagation, IEEE Trans. Antennas Propag., vol. 57, no. 9, pp. 2706-2714, September 2009.

[17] G. Apaydin, O. Ozgun, M. Kuzuoglu, L. Sevgi, Two-way split-step Fourier and finite element based parabolic equation propagation tools: Comparison and calibration, in Proc. IEEE Int. Symp. Antennas Propagation USNC/URSI Nat. Radio Sci. Meet., Toronto, ON, Canada, July $11-17,2010$, pp. 1-4.

[18] O. Ozgun, G. Apaydin, M. Kuzuoglu, L. Sevgi, Two-way Fourier Split Step Algorithm over Variable Terrain with Narraow and Wide Angle Propagators, in Proc. IEEE Int. Symp. Antennas Propagation USNC/URSI Nat. Radio Sci. Meet., Toronto, ON, Canada, July 11-17, 2010, pp. 1-4.

[19] G. Apaydin, O. Ozgun, M. Kuzuoglu, L. Sevgi, A Novel Two-Way FiniteElement Parabolic Equation Groundwave Propagation Tool, Tests With Canonical Structures and Calibration, IEEE Transac. on Geoscience and Remote Sensing, vol. 49, no. 8, pp. 2887-2899, 2011.

[20] A. Fluerasu, C. Letrou, Gaussian Beam Shooting for 3D physical modeling of propagation channels, Annales des tlcommunications - Annals of telecommunications, vol. 64, no. 11-12, pp. 763-776, December 2009.

[21] I. Ghannoum, C. Letrou, G. Beauquet, Gaussian beam shooting algorithm based on iterative frame decomposition, EuCAP 2010: 4th European Conference on Antennas and Propagation, Elsevier B.V. (USB key proceedings), April 12-16, 2010, Barcelone, Spain.

[22] I. Ghannoum, C. Letrou, G. Beauquet, Frame based gaussian beam bouncing, EMTS '10: 20th International Symposium on Electromagnetic Theory, IEEE, 16-19 August 2010, Berlin, Germany, 2010, pp. 68-71, ISBN 978-1-4244-5155-5.

[23] A. Fluerasu, Modélisation de champs dans le domaine spatiotemporel par une méthode de frame de Gabor. Application à la caractérisation du canal indoor millimétrique, Thèse de 1'Université de Marne-la-Vallée, soutenue le 19 Décembre 2003.

[24] I. Ghannoum, Études d'outils de calcul de propagation Radar en milieu complexe (milieu urbain, présence de multi-trajets) par des techniques de Lancer de Faisceaux Gaussiens, Thèse de Doctorat, Télécom \& Management SudParis et Université Pierre et Marie Curie, soutenue le 22 Septembre 2010.

[25] J. M. Darras, T. George et P. Pouliguen, Diffraction by a sharp edge for an electromagnetic field described by summation of Gaussian beams: the Ufimtsev way, Progress in Electromagnetics Research Symposium (PIERS 2012), Moscow, Aug. 2012.

[26] V. Timchenko, E. Heyman, A. Boag, Gaussian beam summation algorithm for ultra wide band indoor channel characterization, Proc. URSI International Symposium on Electromagnetic Theory (EMTS 2010), August 2010, pp.336-339.

[27] M. Katsav, E. Heyman, Gaussian beam summation representation of beam diffraction by an impedance wedge: a 3D electromagnetic formulation within the Physical Optics approximation, IEEE Trans. on Antennas and Propagation, vol. 60, no. 12, pp. 5843-5858, December 2012.

[28] R.F. Harrington, Field computation by Moment methods, Mac Milan, New-York, 1968. 